\title{
FMO1 catalyzes the production of taurine from hypotaurine
}

\section{Sunil Veeravalli', Ian R. Phillips ${ }^{1,2 *}$, Rafael T. Freire ${ }^{3, \pi}$, Dorsa Varshavi³, Jeremy R. Everett ${ }^{3 *}$, and Elizabeth A. Shephard ${ }^{1 *}$}

From the ${ }^{1}$ Department of Structural and Molecular Biology, University College

London, London WC1E 6BT, UK; ${ }^{2}$ School of Biological and Chemical Sciences,

Queen Mary University of London, London E1 4NS, UK; ${ }^{3}$ Medway Metabonomics

Research Group, University of Greenwich, Chatham Maritime, Kent ME4 4TB, UK.

Running title: FMO1 catalyzes the production of taurine from hypotaurine

ๆ Present address: Signal and Information Processing for Sensing Systems, Institute for Bioengineering of Catalonia (IBEC), The Barcelona Institute of Science and Technology, Baldiri Reixac 10-12, 08028 Barcelona, Spain

*To whom correspondence should be addressed: Ian R. Phillips: Department of Structural and Molecular Biology, University College London, London WC1E 6BT, UK; i.r.phillips@ucl.ac.uk; Jeremy R Everett: Medway Metabonomics Research Group, University of Greenwich, Chatham Maritime, Kent ME4 4TB, UK;

j.r.everett@greenwich.ac.uk; Elizabeth A. Shephard: Department of Structural and Molecular Biology, University College London, London WC1E 6BT, UK; e.shephard@ucl.ac.uk. 


\begin{abstract}
Taurine is one of the most abundant amino acids in mammalian tissues. It is obtained from the diet and by de novo synthesis, from cysteic acid or hypotaurine. Despite the discovery in 1954 that the oxygenation of hypotaurine produces taurine, the identification of an enzyme catalyzing this reaction has remained elusive. In large part this is due to the incorrect assignment, in 1962, of the enzyme as a NAD-dependent hypotaurine dehydrogenase. For more than 55 years the literature has continued to refer to this enzyme as such. Here we show, both in vivo and in vitro, that the enzyme that oxygenates hypotaurine to produce taurine is flavin-containing monooxygenase 1 (FMO1). Metabolite analysis of the urine of Fmol-null mice by ${ }^{1} \mathrm{H}$ NMR spectroscopy revealed a build-up of hypotaurine and a deficit of taurine in comparison with the concentrations of these compounds in the urine of wild-type mice. In vitro assays confirmed that FMO1 of human catalyzes the conversion of hypotaurine to taurine utilizing either NADPH or NADH as co-factor. FMO1 has a wide substrate range and is best known as a xenobiotic- or drug-metabolizing enzyme. The identification that the endogenous molecule hypotaurine is a substrate for the FMO1catalyzed production of taurine resolves a long-standing mystery. This finding should help establish the role FMO1 plays in a range of biological processes in which taurine or its deficiency is implicated, including conjugation of bile acids, neurotransmitter, anti-oxidant and anti-inflammatory functions, the pathogenesis of obesity and skeletal muscle disorders.
\end{abstract}




\section{Introduction}

Taurine (2-aminoethanesulfonic acid) is one of the most abundant amino acids in mammalian tissues (1). It is an organic osmolyte involved in cell volume regulation (1) and has a variety of cytoprotective and developmental roles, particularly in neurological and ocular tissues (2). It is also involved in the formation of bile salts (1) and the modulation of intracellular calcium concentration (3). Taurine is considered an essential substance in mammals and its deficiency has been implicated in several pathological conditions; however, the mechanism by which the amino acid exerts its effects is not understood.

Taurine is obtained from the diet and, by de novo synthesis, from cysteic acid (4) or hypotaurine (5). Hypotaurine is itself an organic osmolyte and cytoprotective agent (6) and acts as an anti-oxidant to scavenge highly reactive hydroxyl radicals (7). The oxygenation of hypotaurine to produce taurine was discovered in 1954 (5). Later the enzyme that converts hypotaurine to taurine was reported to be an NADdependent hypotaurine dehydrogenase (8), but was not isolated or characterized. Subsequently, Oja et al. (9) noted that the production of taurine in tissue extracts was optimal at pH 9.0 and was stimulated by oxygenation. These authors concluded that the enzyme that converts hypotaurine to taurine was not an NAD-dependent hypotaurine dehydrogenase. By overlooking the work of Oja et al. (9), and giving credence to the earlier study (8), the enzyme that catalyzes the conversion of hypotaurine to taurine has continued to be reported as a hypotaurine dehydrogenase, utilising NAD as cofactor (EC 1.8.1.3). Because an enzyme that catalyzes the reaction has not been identified or isolated, the conversion of hypotaurine to taurine has sometimes been referred to as non-enzymatic. 
Here we show, both in vivo, through the use of a knockout-mouse line, and in vitro, by assays of human enzymes, that oxygenation of hypotaurine to produce taurine is catalyzed by flavin-containing monooxygenase 1 (FMO1).

\section{Results}

Previous phenotypic analysis (10-13) has identified metabolic differences between mice in which the Fmol, Fmo2 and Fmo4 genes had been deleted (Fmol ${ }^{-/}$, $2^{-/}, 4^{-/}$mice) (10) and wild-type animals. As an extension of this work we have used one-dimensional (1D) ${ }^{1} \mathrm{H}$ NMR spectroscopy to compare the urinary metabolic profiles of the knockout mouse line and wild-type animals. Analysis of the urine of male and female $\mathrm{Fmol}^{-/}, 2^{-/}, 4^{-/-}$mice revealed signals at 2.66 and $3.37 \mathrm{ppm}$, corresponding to those of hypotaurine. Such signals were markedly lower in the urine of wild-type mice (Fig. 1A, B). Signals at 3.28 and $3.43 \mathrm{ppm}$, corresponding to those of taurine, were detected in the urine of both wild-type and $\mathrm{Fmol}^{-/}, 2^{-/-}, 4^{-/}$mice, but their intensities were lower in the latter (Fig. 1A, B). The identities of taurine and hypotaurine in urine samples were confirmed by two-dimensional (2D) NMR (Supporting Information Fig. S1). The urinary ratio of taurine to hypotaurine + taurine was significantly less in $\mathrm{Fmol}^{-/,}, 2^{-/-}, 4^{-/-}$mice than in wild-type mice $(P<0.0001)$

(Fig. 1C). The build-up of hypotaurine and the concomitant decrease of taurine in the urine of $\mathrm{Fmol}^{-/}, 2^{-/}, 4^{-/}$mice (Fig. 1A, B, C) suggests that the formation of taurine from hypotaurine is catalyzed by an FMO.

Of the three genes deleted in the knockout mouse, the gene encoding FMO4 is expressed at very low levels in mouse (14) and human (15), and that encoding FMO2 is expressed in low amounts in mouse (14) and, in most humans, the gene does not encode a functional protein because of the presence of a premature stop codon (16). In 
contrast, the gene encoding FMO1 is relatively highly expressed in a number of tissues in both mouse (14) and human (15). Therefore, in humans the most likely candidate for catalyzing the oxygenation of hypotaurine to produce taurine is FMO1. To investigate whether this was the case, baculosomes containing recombinantly expressed human FMO1 were incubated with hypotaurine and the cofactor NADPH at $\mathrm{pH} 8.5$, the optimum for FMO1, and at the more physiological $\mathrm{pH}$ of 7.4. Analysis of reaction products by $1 \mathrm{D}^{1} \mathrm{H}$ NMR spectroscopy identified signals at 3.276 and 3.433 ppm, corresponding to taurine, at both pHs (Fig. 2A). As expected of an FMO1catalyzed reaction, with $\mathrm{NADPH}$ as cofactor, production of taurine was greater at $\mathrm{pH}$ 8.5 than at $\mathrm{pH} 7.4(P<0.01)($ Fig. $2 \mathrm{~B})$. In comparison, very little taurine was detected in incubations of control insect cell microsomes at $\mathrm{pH} 8.5$ and none at $\mathrm{pH} 7.4$ (Fig. 2A, B). The identity of taurine as a product of FMO1-catalyzed reactions was confirmed by high-resolution UPLC-electrospray mass spectrometry against an authentic reference standard (Fig. S2) (17).

We also investigated whether the conversion of hypotaurine to taurine could be catalyzed by either of the two other major functional FMOs of humans, FMO3 and FMO5 (18). In comparison with baculosomes containing human FMO1, those containing FMO3 produced much lower amounts of taurine at $\mathrm{pH} 8.5$ or 7.4, with either NADPH or NADH as cofactor $(P<0.01)$. Production of taurine by baculosomes containing human FMO5 was significantly less than by FMO3-baculosomes $(P$ $<0.05$ ) and was similar to that produced by control insect cell microsomes (Fig. 2B). These results confirm that production of taurine from hypotaurine can be catalyzed by FMO1 and, to a much smaller extent, by FMO3, but not significantly by FMO5.

The production of taurine in the Fmol-null mice was less affected in females ( $\sim 55 \%$ depletion) than in males $(\sim 70 \%$ depletion) $(P<0.0001)$ (Fig. 1C). This gender 
difference is likely due to the contribution in female Fmol-null mice of FMO3, which is absent from the liver of adult male mice $(14,19)$. In the case of wild-type mice, the presence of FMO1, which is more effective than FMO3 in catalyzing the formation of taurine from hypotaurine, as evidenced by analysis in vitro (Fig. 2B), greatly outweighs the effect of the presence in females of FMO3.

FMOs, despite being termed NADPH-dependent monooxygenases, have been reported to be able also to use NADH as a cofactor (20). We found that in catalyzing the production of taurine from hypotaurine, FMO1 could use either NADPH or NADH as cofactor (Fig. 2B). However, whereas NADPH is the more effective cofactor at $\mathrm{pH} 8.5(P<0.01)$, at $\mathrm{pH} 7.4$ the cofactors are equally effective (Fig. 2B). When NAD was used as cofactor for FMO1 the amount of taurine produced was very low and not significantly different from that produced in the absence of cofactor (Fig. 2B). FMO1 could also use NADH as cofactor for $S$-oxygenation of the FMO model substrate methimazole (Fig. 2C). However, in the case of FMO3, methimazole oxygenation was dependent on NADPH (Fig. 2D).

Kinetic parameters of FMO1-catalyzed oxygenation of hypotaurine to produce taurine were estimated under conditions at which the enzyme was most active: $\mathrm{pH} 8.5$ with NADPH as cofactor (Fig. 2B). ${ }^{1} \mathrm{H}$ NMR spectroscopic analysis revealed that under these conditions there was a 1:1 ratio of taurine production to NADPH oxidation. The kinetics of the enzyme-catalyzed reaction could therefore be assessed by monitoring depletion of NADPH (measured at $\mathrm{A}_{340 \mathrm{~nm}}$ ). A plot of kinetic data is shown in Fig. 3A. Direct linear plots of data (velocity versus substrate concentration) in parameter space gave estimates for $K_{\mathrm{M}}$ of $\sim 4.1 \mathrm{mM}$ and $\mathrm{V}_{\max }$ of $\sim 7.5 \mu \mathrm{M} \mathrm{min}{ }^{-1}$, giving a $k_{\text {cat }}$ of $\sim 55 \mathrm{~min}^{-1}$. Further support for the ability of FMO1 to utilize hypotaurine as a substrate is provided by the finding that hypotaurine, at 
concentrations comparable to the $K_{\mathrm{M}}$ of FMO1-catalyzed hypotaurine oxygenation, acted as an effective competitor of FMO1-catalyzed S-oxygenation of methimazole (Fig. 3B).

\section{Discussion}

We have confirmed both in vivo, by ${ }^{1} \mathrm{H}$ NMR metabolite profiling of the urine of $\mathrm{Fmol}^{-/}, 2^{-/-}, 4^{-/}$mice, and in vitro, by analysis of the catalytic activity of FMOs of humans, that formation of taurine from hypotaurine is catalyzed by FMO1, a monooxygenase, and that the enzyme can utilize either NADPH or NADH as cofactor. Our results from knockout mice show that in the absence of FMO1 most taurine production is abolished in both males and females, suggesting that the major source of this abundant amino acid is the FMO1-catalyzed oxygenation of hypotaurine (Fig. 4). Consistent with this, the lack of production of taurine from hypotaurine in vitro in the absence of enzyme indicates that non-enzymatic conversion does not contribute substantially to taurine production.

FMOs (EC 1.14.13.8) are best known for their role in the metabolism of xenobiotics, including therapeutic drugs (reviewed in (21)) and foreign chemicals such as organophosphate insecticides (reviewed in (22)). Of the members of the FMO family, FMO1 has the broadest substrate range (reviewed in (22)). In addition to its role in xenobiotic metabolism, FMO1 has been identified as a novel regulator of energy balance (12).

In humans, FMO1 is expressed in a range of tissues in which the action of taurine has been implicated, for example, kidney (15), brain (23), small intestine (24), heart (25) and a number of endocrine tissues, including pancreas, adrenal and testis (23). In human liver, FMO1 is expressed in the fetus, but is switched off after birth 
$(15,26,27)$. This pattern of expression is consistent with the decline in taurine concentration in the liver after birth (28). In contrast, in adult rodent liver, the gene encoding FMO1 is highly expressed (14) and taurine is abundant (9).

Concentrations of taurine are high in human and monkey fetal brain, but fall during development (28). The decline in taurine concentration in developing brain is consistent with the decrease in expression of Fmol in mouse brain during development (14). FMO1 is active in mouse brain, as evidenced by its catalysis of the $N$-oxygenation of the tricyclic antidepressant imipramine (10). FMO1 would therefore be expected to contribute to the production in brain of taurine from the precursor hypotaurine.

Taurine deficiency is implicated in a number of pathological conditions, including cardiomyopathy, muscular abnormalities and renal dysfunction (2). Conversely, taurine supplementation has been reported to have positive effects on health, for instance, in lowering total plasma cholesterol (29) and in overcoming insulin resistance (2). Given our finding that FMO1 catalyzes the formation of taurine from hypotaurine it is of interest that Fmol-null mice exhibit some characteristics in common with those of taurine deficiency: raised plasma concentrations of cholesterol (13) and glucose (12).

The $K_{\mathrm{M}}$ of FMO1 for hypotaurine is similar to that of cysteine dioxygenase of human, an upstream enzyme in the taurine biosynthetic pathway (Fig. 4), for its substrate cysteine (30). Although the $K_{\mathrm{M}}$ for the FMO1-catalyzed oxygenation of hypotaurine is high, our results from the knockout-mouse line indicate that FMO1 is physiologically relevant for the production of taurine.

In addition to Fmol, two other genes are deleted in the knockout mouse line, Fmo2 and Fmo4. Most humans are homozygous for a nonsense mutation of FMO2, 
c. $1414 \mathrm{C}>\mathrm{T}[\mathrm{p} .(\mathrm{G} \ln 472 *)]$, the $F M O 2 * 2 A$ allele, and do not express functional FMO2 (16,31), and in the case of FMO4 the gene is expressed at very low levels (15). Thus, although we cannot eliminate the possibility that FMO2 or FMO4 are able to catalyze hypotaurine oxygenation in vitro, neither of these enzymes is likely to contribute substantially to the production of taurine in humans in vivo.

Commensurate with a role for FMO1 in endogenous metabolism, the gene contains few non-synonymous polymorphisms (32), each of which is present at very low frequency (33) and only one has a significant, but substrate-dependent, effect on catalytic activity (34). However, inter-individual variation of up to 5-fold in the expression of FMOI in adult human tissues such as kidney (35) and small intestine (24) could affect taurine production and thus contribute to an intracellular deficiency of the amino acid. In addition, the involvement of FMO1 in the metabolism of drugs implies that drug substrates of the enzyme would compete with hypotaurine for available enzyme and thus compromise taurine production, leading to potentially adverse effects on general health and therapeutic response.

\section{Experimental procedures}

Animals: All mice used in this study were bred at University College London. The $\mathrm{Fmol}^{-/}, 2^{-/-}, 4^{-/-}$mouse line was constructed as described previously $(10,36)$. Mice were genotyped as described (37). Mice were given free access to food (a standard chow diet, Teklad Global 18\% ProteinRodent Diet, Harlan Laboratories, Inc., Madison, WI) and water. Animal procedures were carried out in accordance with the UK Animal Scientific Procedures Act and with local ethics committee approval (Animal Welfare and Ethical Review Body) and appropriate Home Office Licenses. Urine was collected between 10:00 AM and 12:00 PM (noon) from male and female 
mice aged 15-16 weeks. Urine samples were immediately frozen on solid $\mathrm{CO}_{2}$ and stored at $-80{ }^{\circ} \mathrm{C}$ until analyzed by ${ }^{1} \mathrm{H}$ NMR spectroscopy, as described below.

Enzyme assays: All reaction mixtures (final volumes of $250 \mu 1$ in a Corning Costar 96-well cell-culture plate, VWR, Lutterworth, Leicestershire, UK) were incubated in a Sunrise absorbance microplate reader (Tecan, Grödig, Austria) equipped with Magellan software, v. 6.2. Reaction mixtures contained either $0.1 \mathrm{M}$ potassium phosphate buffer, $\mathrm{pH} 7.4$, or $0.1 \mathrm{M}$ Tris-HCl, $\mathrm{pH} 8.5,1$ mM EDTA (aerated immediately before use by shaking for $5 \mathrm{~min}$ at room temperature), $2.5 \mathrm{mM}$ hypotaurine, $0.5 \mathrm{mM}$ NADPH, NADH or NAD, or no cofactor, and baculosomes containing human FMO1, FMO3 or FMO5 (135 nM final concentration) (Sigma Aldrich, Gillingham, Dorset, UK) or an equivalent amount of control insect cell microsomes (Corning Life Sciences, Woburn, MA). The mixtures were incubated at $37{ }^{0} \mathrm{C}$ for $60 \mathrm{~min}$. Monitoring of NADPH or NADH depletion, measured at $\mathrm{A}_{340 \mathrm{~nm}}$, revealed that reaction velocities were linear over the 60-min period. Samples were stored at $-80{ }^{\circ} \mathrm{C}$ until analysed by ${ }^{1} \mathrm{H}$ NMR spectroscopy, as described below.

Methimazole $S$-oxygenation was measured by the method of Dixit and Roche (38). Reaction mixtures contained final concentrations of $67 \mathrm{nM}$ of human FMO1 or FMO3 in baculosomes (Sigma Aldrich), $2.5 \mathrm{mM}$ methimazole and $0.5 \mathrm{mM}$ NADPH or $\mathrm{NADH}$, and were incubated at $37^{\circ} \mathrm{C}$.

For estimation of kinetic parameters, reaction mixtures were assembled, by adding, to final concentrations, in the following order $0.1 \mathrm{M}$ Tris- $\mathrm{HCl}(\mathrm{pH} 8.5), 1 \mathrm{mM}$ EDTA (aerated immediately before use by shaking at $37^{\circ} \mathrm{C}$ for $10 \mathrm{~min}$ ), $0.5 \mathrm{mM}$ NADPH and baculosomes containing human FMO1 (135 nM final concentration) (Sigma Aldrich). The mixtures were equilibrated at $37{ }^{\circ} \mathrm{C}$ for $3 \mathrm{~min}$, to allow 
formation of the active C4a-peroxyflavin species of the FAD prosthetic group of

FMO. Reactions were initiated by addition of hypotaurine, to final concentrations of 1

to $10 \mathrm{mM}$ or, in the case of blank samples, an equivalent volume of buffer was added.

Reaction mixtures were incubated at $37{ }^{\circ} \mathrm{C}$. The initial velocity of enzyme-catalyzed

reactions was assessed by monitoring the depletion of NADPH, measured at $\mathrm{A}_{340 \mathrm{~nm} .} \Delta$

$\mathrm{A}_{340 \mathrm{~nm}}$ was converted to $\triangle \mathrm{NADPH}$ using a molar extinction coefficient of $6.2 \times 10^{3}$

$\mathrm{M}^{-1} \mathrm{~cm}^{-1}$ and a light-path length of $0.73 \mathrm{~cm}$. To determine substrate (hypotaurine)-

dependent oxygenation of NADPH, readings from a blank sample (a reaction mixture containing no hypotaurine) were subtracted.

The ability of hypotaurine to act as a competitor substrate of FMO1 was assessed by measuring the effect of various concentrations of hypotaurine on FMO1-catalyzed $S$-oxygenation of methimazole. Methimazole $S$-oxygenation was measured by the method of Dixit and Roche (38), as described above, in reaction mixtures containing final concentrations of $67 \mathrm{nM}$ human FMO1 in baculosomes (Sigma Aldrich), $4 \mu \mathrm{M}$ methimazole and 2.5 to $100 \mathrm{mM}$ hypotaurine.

Sample preparation for NMR spectroscopy: Urine samples $(50 \mu 1)$ were prepared for NMR spectroscopy as described previously (39). Enzyme assay samples were thawed, vortexed, then $160 \mu \mathrm{l}$ of sample was mixed with $80 \mu \mathrm{l}$ of $0.6 \mathrm{M}$ phosphate buffer, as described previously (39). The samples were re-vortexed and centrifuged at $13000 \mathrm{~g}$ for $5 \mathrm{~min}$ at $4{ }^{0} \mathrm{C}$. Supernatant $(200 \mu \mathrm{l})$ was then pipetted into $3.0-\mathrm{mm}$ outer diameter (o.d.) SampleJet NMR tubes (Norell, S-3.0-500-1).

NMR spectroscopic analysis: ${ }^{1} \mathrm{H}$ NMR spectra of urine and enzyme assay samples were recorded on a Bruker Avance III spectrometer (Bruker BioSpin GmbH, 
Rheinstetten, Germany) operating at $600.44 \mathrm{MHz}$ and at a temperature of $300.0 \mathrm{~K}$, using a standard 1D NOESY presaturation pulse sequence with gradient pulses (noesygppr1d), as described previously (39).

NMR spectral processing was carried out in MNova (MestReNova, version 12.0.1-20560, Mestrelab Research S.L.). The deconvolution of the peaks for metabolite quantification was done using the MNova GSD algorithm. The peak areas were obtained, and the residuals were manually minimized by adjusting the fitting parameters of each peak. Data were imported into Matlab (R2014b, MathWorks). Statistical analysis was performed using an unpaired two-tailed t-test. Significance level $P<0.05$.

NMR data deposition: Original NMR data will be deposited in MetaboLights (EBI UK) (40) after publication.

Metabolite identification: NMR-based metabolite identification was carried out using standard methods, as described (17), and using information from the literature and public databases including the Human Metabolite Database (41) (HMDB, http://www.hmdb.ca/).2018). Hypotaurine in $\mathrm{Fmol}^{-/}, 2^{-/-}, 4^{-/-}$mouse urine showed the following features: $2.665(\mathrm{t}, 6.9 \mathrm{~Hz}), 58.6$ (HSQC) with HMBC to 36.4 and COSY (see Fig. S1) to 3.365 (t, $6.9 \mathrm{~Hz}$ ), 36.5 (HSQC) and HMBC to $58.5 \mathrm{ppm}$, in complete agreement with literature values: $2.66(\mathrm{t}, 6.9 \mathrm{~Hz}), 58.5$ and $3.35(\mathrm{t}, 6.9 \mathrm{~Hz}), 36.2 \mathrm{ppm}$ (HMDB00965, accessed from http://www.hmdb.ca/spectra/nmr_one_d/1626 on 5 February 2019). Taurine in $\mathrm{Fmol}^{-/-}, 2^{-/-}, 4^{-/}$and wild-type mouse urine showed the following features: $3.283(\mathrm{t}, 6.6 \mathrm{~Hz}), 50.6$ (HSQC) with HMBC to 38.3 and COSY to $3.433(\mathrm{t}, 6.6 \mathrm{~Hz}), 38.4$ (HSQC) and HMBC to $50.5 \mathrm{ppm}$, in complete agreement with 
literature values: 3.25 (t, $6.6 \mathrm{~Hz}), 50.4$ and 3.42 (t, $6.6 \mathrm{~Hz}), 38.3 \mathrm{ppm}$

(HMDB0000251, accessed from http://www.hmdb.ca/spectra/nmr_one d/1277 on 5th

February 2019). Both hypotaurine and taurine in the urine samples were

unambiguously identified using the recent MICE criteria (42).

Acknowledgments: We thank Mr Mohamed Said and Ms Dorna Varshavi for assistance with NMR spectroscopy and Dr Iain Goodall for assistance with UPLCMS. We also thank Professors Jeremy Nicholson and Elaine Holmes for access to NMR facilities at Imperial College London.

Conflict of interest: The authors declare that they have no conflicts of interest with the contents of this article.

Author contributions: a complete list of contributions to the paper;

Conceptualization (IRP, JRE, EAS)

Data Curation (SV, IRP, RTF, DV, JRE, EAS, )

Formal analysis (SV, IRP, RTF, JRE, EAS)

Investigation (SV, IRP, EAS, RTF, DV, JRE)

Methodology (IRP, EAS, JRE)

Project administration (IRP, EAS, JRE)

Resources (IRP, EAS, JRE)

Software (RTF, DV)

Supervision (IRP, JRE, EAS) 
Validation (SV, IRP, RTF, DV, JRE, EAS)

Visualization (SV, IRP, EAS, RTF, JRE)

Writing - original draft (IRP, EAS)

Writing - review \& editing (SV, IRP, RTF, DV, JRE, EAS)

\section{Data and materials availability:}

NMR Spectroscopic data will be deposited in MetaboLights (EBI) following acceptance of the manuscript. 


\section{References}

1. Huxtable, R. J. (1992) Physiological actions of taurine. Physiol. Rev. 72, 10163

2. Ripps, H., and Shen, W. (2012) Review: taurine: a "very essential” amino acid. Mol. Vis. 18, 2673-2686

3. Lombardini, J. B. (1983) Effects of ATP and taurine on calcium uptake by membrane preparations of the rat retina. J. Neurochem. 40, 402-406

4. Edgar, S. E., Hickman, M. A., Marsden, M. M., Morris, J. G., and Rogers, Q. R. (1994) Dietary cysteic acid serves as a precursor of taurine for cats. J. Nutr. 124, 103-109

5. Cavallini D., De Marco, C., Mondovi, B., and Stirpe, F. (1954) The biological oxidation of hypotaurine. Biochim. Biophys. Acta. 15, 301-303

6. Yancey, P. H. (2005) Organic osmolytes as compatible, metabolic and counteracting cytoprotectants in high osmolarity and other stresses. $J$. Exp. Biol. 208, 2819-2830

7. Aruoma, O. I., Halliwell, B., Hoey, B. M., and Butler, J. (1988) The antioxidant action of taurine, hypotaurine and their metabolic precursors. Biochem. J. 256, 251-255

8. Sumizu, K. (1962) Oxidation of hypotaurine in rat liver. Biochim. Biophys. Acta. 63, 210-212

9. Oja, S. S., and Kontro, P. (1981) Oxidation of hypotaurine in vitro by mouse liver and brain tissues. Biochim. Biophys. Acta - Gen. Subj. 677, 350-357

10. Hernandez, D., Janmohamed, A., Chandan, P., Omar, B. A., Phillips, I. R., and Shephard, E. A. (2009) Deletion of the mouse Fmol gene results in enhanced pharmacological behavioural responses to imipramine. Pharmacogenet. 
Genomics. 19, 289-299

11. Shephard, E. A., and Phillips, I. R. (2010) The potential of knockout mouse lines in defining the role of flavin-containing monooxygenases in drug metabolism. Expert Opin. Drug Metab. Toxicol. 6, 1083-1094

12. Veeravalli, S., Omar, B. A., Houseman, L., Hancock, M., Gonzalez Malagon, S. G., Scott, F., Janmohamed, A., Phillips, I. R., and Shephard, E. A. (2014) The phenotype of a flavin-containing monooyxgenase knockout mouse implicates the drug-metabolizing enzyme FMO1 as a novel regulator of energy balance. Biochem. Pharmacol. 90, 88-95

13. Veeravalli, S., Karu, K., Scott, F., Fennema, D., Phillips, I. R., and Shephard, E. A. (2018) Effect of flavin-containing monooxygenase genotype, mouse strain, and gender on trimethylamine $N$-oxide production, plasma cholesterol concentration, and an index of atherosclerosis. Drug Metab. Dispos. 46, 20-25

14. Janmohamed, A., Hernandez, D., Phillips, I. R., and Shephard, E. A. (2004) Cell-, tissue-, sex- and developmental stage-specific expression of mouse flavin-containing monooxygenases (Fmos). Biochem. Pharmacol. 68, 73-83

15. Dolphin, C. T., Cullingford, T. E., Shephard, E. A., Smith, R. L., and Phillips, I. R. (1996) Differential developmental and tissue-specific regulation of expression of the genes encoding three members of the flavin-containing monooxygenase family of man, FMO1, FMO3 and FM04. Eur. J. Biochem. 235, 683-689

16. Dolphin, C. T., Beckett, D. J., Janmohamed, A., Cullingford, T. E., Smith, R. L., Shephard, E. A., and Phillips, I. R. (1998) The flavin-containing monooxygenase 2 gene (FMO2) of humans, but not of other primates, encodes a truncated, nonfunctional protein. J. Biol. Chem. 273, 30599-30607 
17. Dona, A. C., Kyriakides, M., Scott, F., Shephard, E. A., Varshavi, D., Veselkov, K., and Everett, J. R. (2016) A guide to the identification of metabolites in NMR-based metabonomics/metabolomics experiments. Comput. Struct. Biotechnol. J. 14, 135-53

18. Overby, L. H., Carver, G. C., and Philpot, R. M. (1997) Quantitation and kinetic properties of hepatic microsomal and recombinant flavin-containing monooxygenases 3 and 5 from humans. Chem. Biol. Interact. 106, 29-45

19. Falls, J. G., Blake, B. L., Cao, Y., Levi, P. E., and Hodgson, E. (1995) Gender differences in hepatic expression of flavin-containing monooxygenase isoforms (FMO1, FMO3, and FMO5) in Mice. J. Biochem. Toxicol. 10, 171-177

20. Hvattum, E., Bergseth, S., Pedersen, C. N., Bremer, J., Aarsland, A., and Berge, R. K. (1991) Microsomal oxidation of dodecylthioacetic acid (a 3-thia fatty acid) in rat liver. Biochem. Pharmacol. 41, 945-953

21. Phillips, I. R., and Shephard, E. A. (2017) Drug metabolism by flavincontaining monooxygenases of human and mouse. Expert Opin. Drug Metab. Toxicol. 13, 167-181

22. Krueger, S. K., and Williams, D. E. (2005) Mammalian flavin-containing monooxygenases: structure/function, genetic polymorphisms and role in drug metabolism. Pharmacol. Ther. 106, 357-387

23. Hernandez, D., Janmohamed, A., Chandan, P., Phillips, I. R., and Shephard, E. A. (2004) Organization and evolution of the flavin-containing monooxygenase genes of human and mouse: identification of novel gene and pseudogene clusters. Pharmacogenetics. 14, 117-130

24. Yeung, C. K., Lang, D. H., Thummel, K. E., and Rettie, A. E. (2000) Immunoquantitation of FMO1 in human liver, kidney, and intestine. Drug 
Metab. Dispos. 28, 1107-1111

25. Kim, Y. H., Lim, D. S., Lee, J. H., Lim, D.-S., Shim, W. J., Ro, Y. M., Park, G. H., Becker, K. G., Cho-Chung, Y. S., and Kim, M.-K. (2003) Gene expression profiling of oxidative stress on atrial fibrillation in humans. Exp. Mol. Med. 35, $336-49$

26. Dolphin, C., Shephard, E. A., Povey, S., Palmer, C. N. A., Ziegler, D. M., Ayesh, R., Smith, R. L., and Phillips, I. R. (1991) Cloning, primary sequence, and chromosomal mapping of a human flavin-containing monooxygenase (FMO1). J. Biol. Chem. 266, 12379-12385

27. Koukouritaki, S. B., Simpson, P., Yeung, C. K., Rettie, A. E., and Hines, R. N. (2002) Human hepatic flavin-containing monooxygenases 1 (FMO1) and 3 (FMO3) developmental expression. Pediatr. Res. 51, 236-243

28. Sturman, J. A., and Gaull, G. E. (1975) Taurine in the brain and liver of the developing human and monkey. J. Neurochem. 25, 831-835

29. Murakami, S. (2015) Role of taurine in the pathogenesis of obesity. Mol. Nutr. Food Res. 59, 1353-1363

30. Ye, S., Wu, X., Wei, L., Tang, D., Sun, P., Bartlam, M., and Rao, Z. (2007) An insight into the mechanism of human cysteine dioxygenase. J. Biol. Chem. 282, $3391-3402$

31. Whetstine, J. R., Yueh, M.-F., Hopp, K. A., McCarver, D. G., Williams, D. E., Park, C.-S., Kang, J. H., Cha, Y.-N., Dolphin, C. T., Shephard, E. A., Phillips, I. R., and Hines, R. N. (2000) Ethnic differences in human flavin-containing monooxygenase 2 (FMO2) polymorphisms: Detection of expressed protein in African-Americans. Toxicol. Appl. Pharmacol. 168, 216-224

32. Phillips, I. R., Francois, A. A., and Shephard, E. A. (2007) The flavin- 
containing monoooxygenases (FMOs): Genetic variation and its consequences for the metabolism of therapeutic drugs. Curr. Pharmacogenomics. 5, 292-313

33. Furnes, B., Feng, J., Sommer, S. S., and Schlenk, D. (2003) Identification of novel variants of the flavin-containing monooxygenase gene family in African Americans. Drug Metab. Dispos. 31, 187-193

34. Furnes, B., and Schlenk, D. (2004) Evaluation of xenobiotic $N$ - and $S$-oxidation by variant flavin-containing monooxygenase 1 (FMO1) enzymes. Toxicol. Sci. 78, 196-203

35. Krause, R. J. (2003) Human kidney flavin-containing monooxygenases and their potential roles in cysteine $S$-conjugate metabolism and nephrotoxicity. $J$. Pharmacol. Exp. Ther. 304, 185-191

36. Hernandez, D., Chandan, P., Janmohamed, A., Phillips, I. R., and Shephard, E. A. (2006) Deletion of genes from the mouse genome using Cre/loxP technology. Methods Mol. Biol. 320, 307-19

37. Hernandez, D., Melidoni, A. N., Phillips, I. R., and Shephard, E. A. (2006) Microinjection of targeted embryonic stem cells and establishment of knockout mouse lines for Fmo genes. Methods Mol. Biol. 320, 329-41

38. Dixit, A., and Roche, T. E. (1984) Spectrophotometric assay of the flavincontaining monooxygenase and changes in its activity in female mouse liver with nutritional and diurnal conditions. Arch. Biochem. Biophys. 233, 50-63

39. Varshavi, D., Scott, F. H., Varshavi, D., Veeravalli, S., Phillips, I. R., Veselkov, K., Strittmatter, N., Takats, Z., Shephard, E. A., and Everett, J. R. (2018) Metabolic biomarkers of ageing in C57BL/6J wild-type and flavincontaining monooxygenase 5 (FMO5)-knockout mice. Front. Mol. Biosci. 5, 116 
40. Haug, K., Salek, R. M., Conesa, P., Hastings, J., de Matos, P., Rijnbeek, M., Mahendraker, T., Williams, M., Neumann, S., Rocca-Serra, P., Maguire, E., González-Beltrán, A., Sansone, S.-A., Griffin, J. L., and Steinbeck, C. (2013) MetaboLights--an open-access general-purpose repository for metabolomics studies and associated meta-data. Nucleic Acids Res. 41, D781-6

41. Wishart, D. S., Feunang, Y. D., Marcu, A., Guo, A. C., Liang, K., VázquezFresno, R., Sajed, T., Johnson, D., Li, C., Karu, N., Sayeeda, Z., Lo, E., Assempour, N., Berjanskii, M., Singhal, S., Arndt, D., Liang, Y., Badran, H., Grant, J., Serra-Cayuela, A., Liu, Y., Mandal, R., Neveu, V., Pon, A., Knox, C., Wilson, M., Manach, C., and Scalbert, A. (2018) HMDB 4.0: the human metabolome database for 2018. Nucleic Acids Res. 46, D608-D617

42. Everett, J. R. (2015) A new paradigm for known metabolite identification in metabonomics/metabolomics: metabolite identification efficiency. Comput. Struct. Biotechnol. J. 13, 131-144 


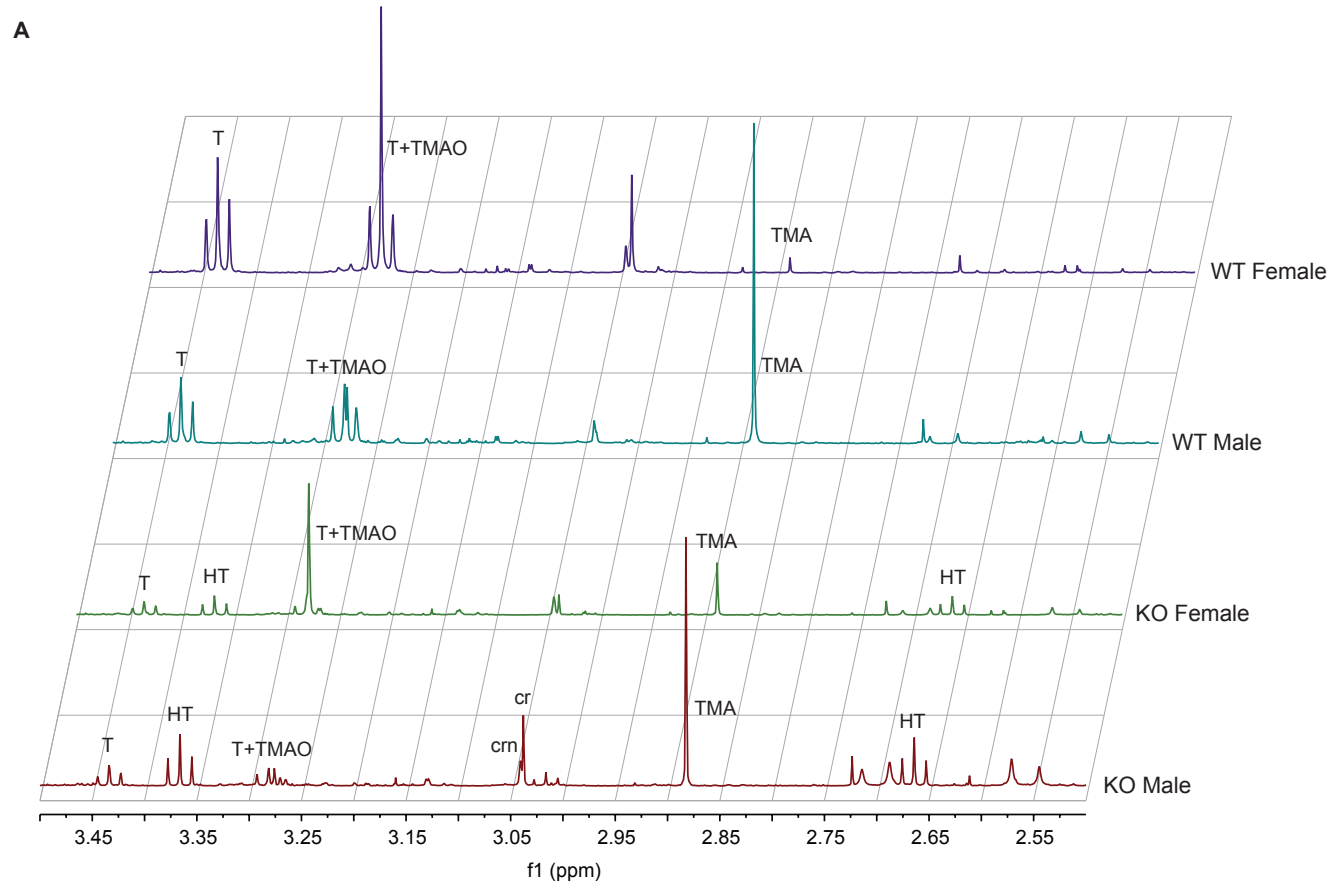

B

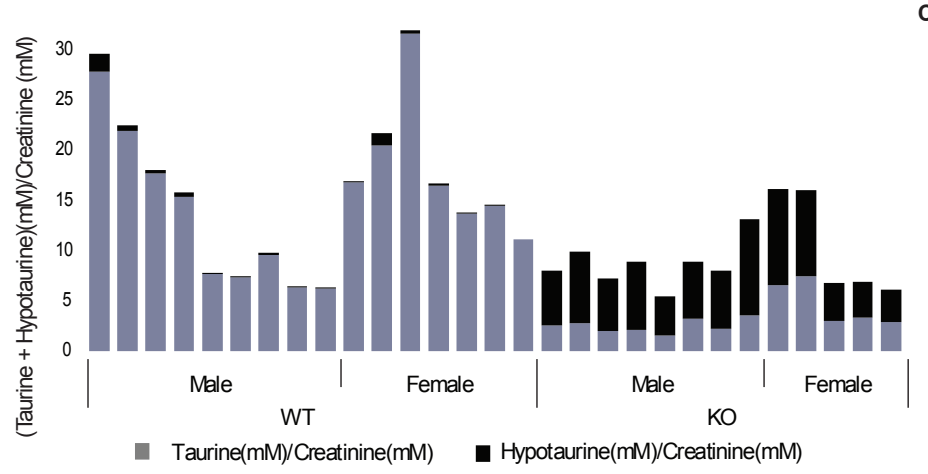

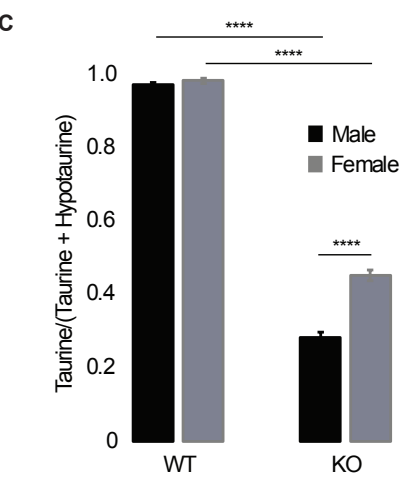

Figure 1. Abundance of hypotaurine and taurine in mouse urine. (A) Representative NMR spectra of urine from $\mathrm{Fmol}^{-/}, 2^{--\alpha}, 4^{-/}(\mathrm{KO})$ male and female mice and wild-type (WT) male and female mice. T, taurine; HT, hypotaurine; TMAO, trimethylamine $N$-oxide; TMA, trimethylamine; Crn, creatinine; Cr, creatine. (B) Proportion of taurine and hypotaurine (normalized to creatinine) in urine from individual male and female WT and KO mice. (C) Average ratios of taurine to taurine + hypotaurine in urine of male and female WT and KO mice. Data are expressed as means \pm SEM $(\mathrm{n}=7-9$, WT; 5-8, KO). ****, $P<0.0001$. 


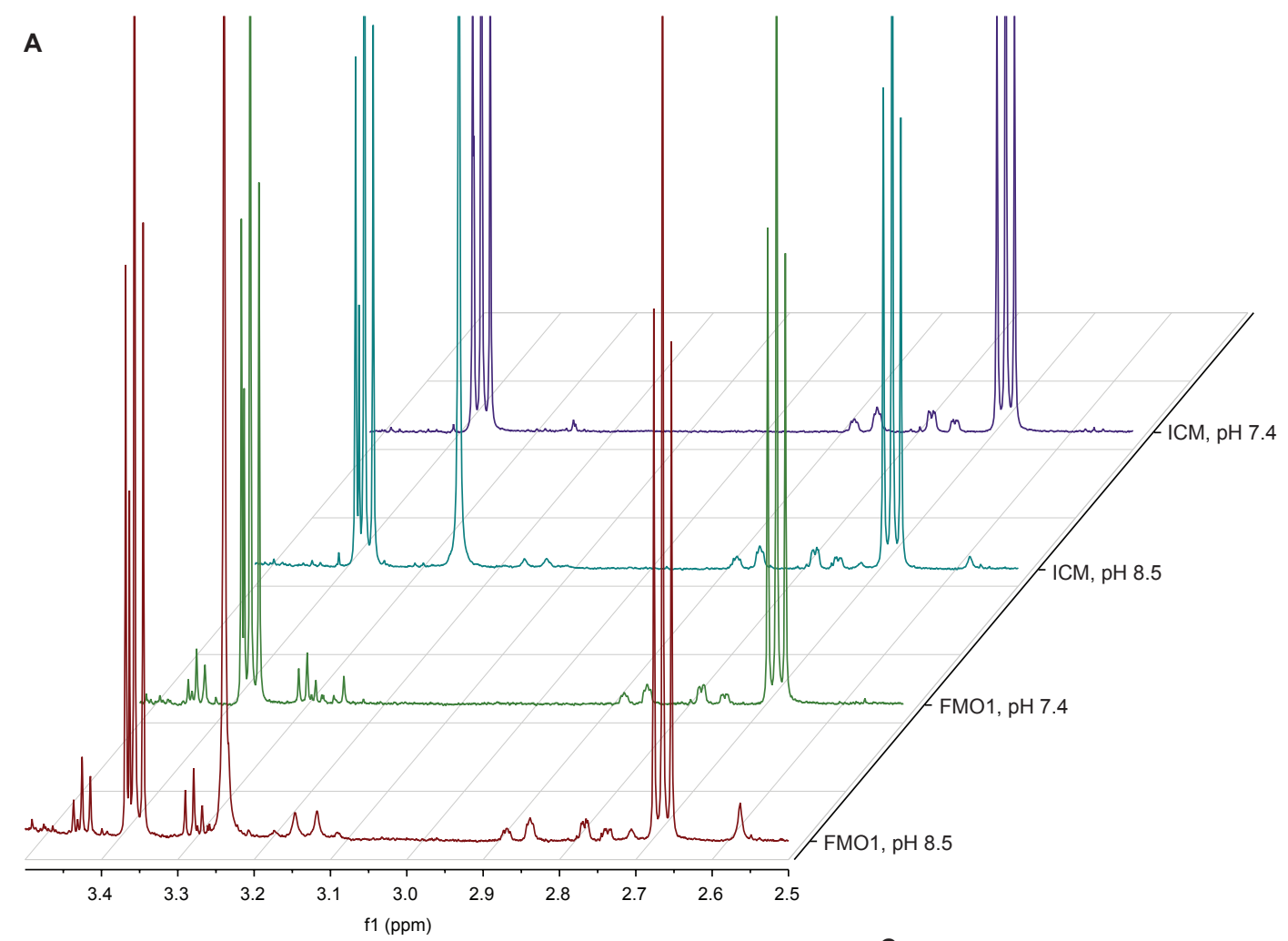

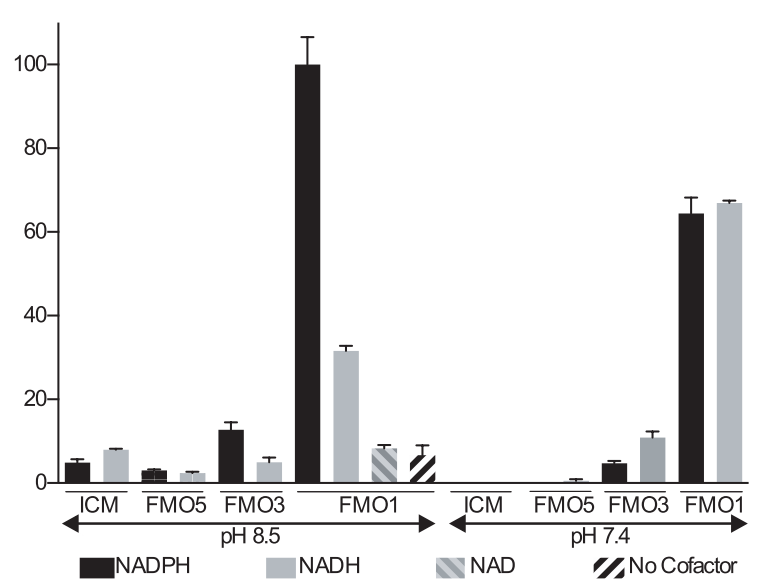

C
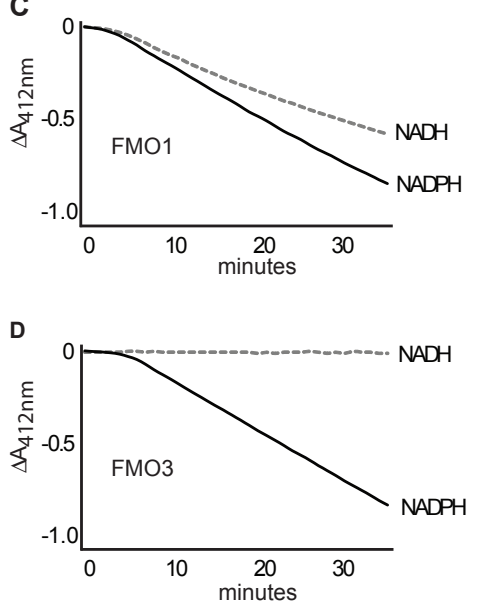

Figure 2. Analysis of in vitro enzyme-catalyzed reactions. (A) Representative NMR spectra of products of reactions catalyzed by baculosomes containing human FMO1 or by insect cell microsomes (ICM) at $\mathrm{pH} 8.5$ or 7.4 . Note: the singlet signal at ca 3.364 ppm overlapping the triplet due to hypotaurine is due to methanol. T, taurine; HT, hypotaurine. (B) Production of taurine from hypotaurine in reactions catalyzed by human FMO1, FMO3 or FMO5 or by ICM, at $\mathrm{pH} 8.5$ or 7.4 , with NADPH or NADH as cofactor. In the case of reactions catalyzed by FMO1 at $\mathrm{pH} 8.5, \mathrm{NAD}$ or no cofactor were also used. Taurine production was quantified 

available under aCC-BY-NC-ND 4.0 International license.

by NMR and is plotted relative to that produced by FMO1-containing baculosomes at $\mathrm{pH} 8.5$ with NADPH as cofactor (set at $100 \%)$. Data are expressed as means $\pm 0.5 \mathrm{x}$ range $(\mathrm{n}=2) .(\mathrm{C}$,

D) Progress curves of methimazole $S$-oxygenation catalyzed by human FMO1 (C) or FMO3

(D), at $\mathrm{pH} 8.5$, with $\mathrm{NADPH}$ or $\mathrm{NADH}$ as cofactor. Reactions were monitored at $\mathrm{A}_{412 \mathrm{~nm}}$. 
A

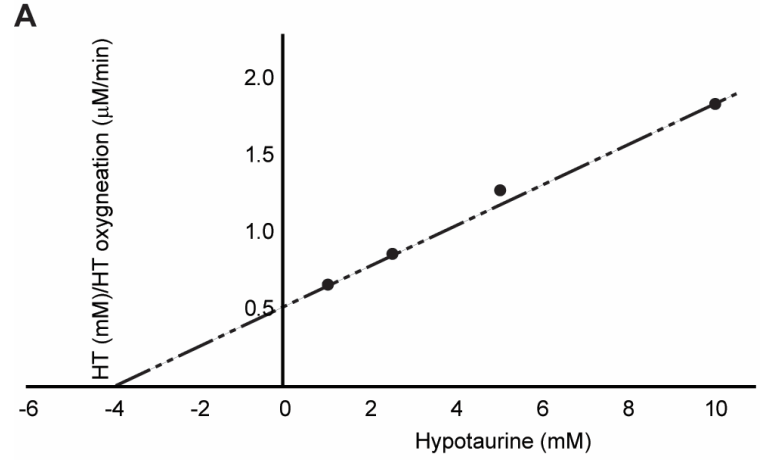

B

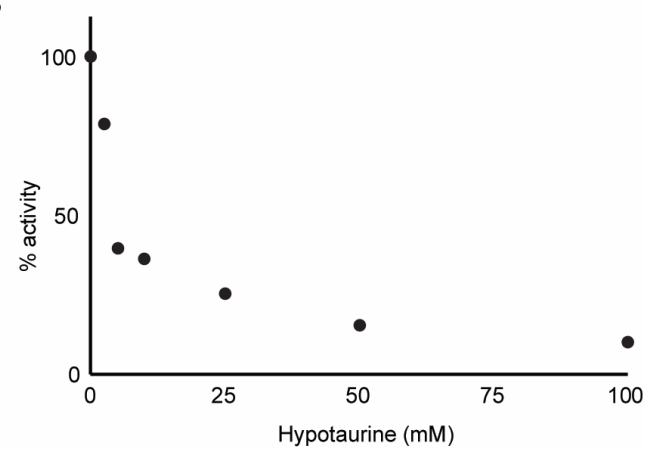

Figure. 3. Kinetic and competition analysis of FMO1-catalyzed reactions. (A) Kinetic analysis of FMO1-catalyzed oxygenation of hypotaurine. Reaction mixtures contained human FMO1, NADPH and various concentrations of hypotaurine. Initial velocity was measured by monitoring hypotaurine-dependent depletion of NADPH at $\mathrm{A}_{340 \mathrm{~nm} .}$ (B) Competition of FMO1-catalyzed $S$-oxygenation of methimazole by hypotaurine. Reaction mixtures contained human FMO1, methimazole, NADPH and various concentrations of hypotaurine. The concentration of methimazole was $50 \%$ of the $K_{\mathrm{M}}$ of FMO1 for this substrate. Initial velocity was measured as described in Experimental Procedures. Methimazole $S$-oxygenation activity was plotted as a percentage of that in the absence of hypotaurine. 
Figure 4. Terminal steps of the biosynthetic pathway of taurine from cysteine. The final reaction in the pathway is catalyzed by FMO1, using either NADPH or NADH as cofactor. CDO, cysteine dioxygenase; CSD, cysteine sulfinate decarboxylase; FMO1, flavin-containing monooxygenase 1 . 